Itinéraires Itinéraires

Littérature, textes, cultures

\title{
Le chapitre face au collage et au montage : Dos Passos, Burroughs, Ballard
}

Chapter Faced with Collage and Montage: Dos Passos, Burroughs, Ballard

\section{Clémentine Hougue}

\section{(2) OpenEdition}

1 Journals

Édition électronique

URL : http://journals.openedition.org/itineraires/7403

DOI : $10.4000 /$ itineraires. 7403

ISSN : 2427-920X

Éditeur

Pléiade

\section{Référence électronique}

Clémentine Hougue, "Le chapitre face au collage et au montage : Dos Passos, Burroughs, Ballard », Itinéraires [En ligne], 2020-1 | 2020, mis en ligne le 01 septembre 2020, consulté le 13 novembre 2020. URL : http://journals.openedition.org/itineraires/7403; DOI : https://doi.org/10.4000/itineraires.7403

Ce document a été généré automatiquement le 13 novembre 2020.

\section{(c) (i) (2)}

Itinéraires est mis à disposition selon les termes de la licence Creative Commons Attribution - Pas d'Utilisation Commerciale - Pas de Modification 4.0 International. 


\title{
Le chapitre face au collage et au montage : Dos Passos, Burroughs, Ballard
}

\author{
Chapter Faced with Collage and Montage: Dos Passos, Burroughs, Ballard
}

\author{
Clémentine Hougue
}

1 Issus des avant-gardes du début du $\mathrm{xx}^{\mathrm{e}}$ siècle, le collage et le montage deviennent progressivement des procédés littéraires à part entière. Si les deux pratiques consistent à combiner des éléments textuels hétérogènes, elles couvrent néanmoins des champs esthétiques sensiblement différents. En effet, le terme de montage, emprunté au cinéma, suppose une articulation, c'est-à-dire une mise en relation entre les éléments : il revêt ainsi une "fonction syntaxique " qui assure la liaison entre les différents fragments montés (Aumont 1983: 47-49). En revanche le collage, issu des arts plastiques fixes (peinture, dessin, sculpture, etc.) repose sur un processus d'agglomération, sans mise en relation des éléments hétérogènes qui composent le texte: cette technique procède d'« une sélection et [d']une combinaison, comme n'importe quel message, mais avec la double condition constitutive de s'emprunter dans des ensembles déjà organisés et de se disposer en un système d'allotopies " (Dubois, Dubois, Édeline, Klinkenberg et Minguet 1978: 14). Ainsi, quand le montage permet une jonction des différents fragments, le collage conserve la disjonction des éléments et maintient leur hétérogénéité. Comme nous le verrons plus loin, la structure globale des œuvres de Dos Passos et Ballard est régie par le montage, quand l'écriture de Burroughs, appelée cutup, relève plus strictement du collage. En outre, Dos Passos utilise localement le collage dans certains chapitres.

2 Il n'en demeure pas moins que les deux pratiques ont en commun de reposer sur un agencement de matières textuelles hétérogènes, en vue de créer une esthétique de la rupture. Que devient le chapitre dans des textes fragmentés par ces procédés? Le changement de chapitre marque habituellement une rupture dans la narration, dans la mesure où il crée des "plages intersticielles dans le continu narratif» (Dionne 2000 : 261) : comment le collage et le montage y articulent-ils leur propre rupture? Les trois 
auteurs que nous nous proposons d'étudier permettent d'apporter à cette question des éléments de réponse à la fois esthétiques et politiques.

John Dos Passos (1896-1970), William S. Burroughs (1914-1997) et James Graham Ballard (1930-2009) s'inscrivent dans une histoire anglo-saxonne du montage et du collage romanesques, voire une histoire nord-américaine (Cran 2014) : bien que Ballard soit britannique, son œuvre est très largement imprégnée de culture américaine, dont les figures populaires (comme Marilyn Monroe ou John Fitzgerald Kennedy) sont des motifs récurrents. Si leurs pratiques s'inscrivent dans un processus historique, c'est aussi dans la mesure où chaque auteur a revendiqué l'influence du précédent (Burroughs affirmant le rôle pionnier de Dos Passos et Ballard revendiquant lui-même l'influence de Burroughs). Se dessine alors une sorte de généalogie du collage romanesque anglo-américain. Ces trois auteurs ont en outre en commun d'articuler profondément critique sociale et déconstruction textuelle, l'invention formelle que constitue l'intervention du collage et du montage dans leur écriture se mettant au service d'un discours critique sur la société de leur époque, notamment sur les images qu'elle produit. Dans leurs œuvres, le chapitre, combiné au collage-montage, apparaît comme un moyen d'interroger les médias (presse écrite, radio, télévision, cinéma) et révèle ainsi la dimension idéologique de l'agencement romanesque.

\section{Intrusions du montage et du collage dans la structure romanesque}

\section{Montage et collage dans U.S.A. de John Dos Passos}

4 La trilogie U.S.A. propose une tentative de définition de la société américaine et de ses mutations, de 1898 à 1927. Le premier roman, 42nd Parallel (1930), évoque la montée du syndicalisme et du socialisme avant la participation américaine à la Première Guerre mondiale en 1917. Le deuxième, 1919 (1932), narre cette même guerre du point de vue américain ; son caractère central dans la trilogie s'articule avec un moment de bascule idéologique : la désillusion des espoirs socialistes. The Big Money (1936), dernier opus de la trilogie, raconte le bouillonnement culturel et l'essor économique des Roaring Twenties, à laquelle la Grande Dépression, qui suit le krach boursier de 1929, mettra brutalement fin.

5 Publiés en un volume en 1938, les trois romans sont divisés en quatre types de chapitres, dont l'énonciation et la narration sont différentes et s'enchaînent de façon plus ou moins régulière tout au long de la trilogie. Le premier type raconte l'histoire de douze personnages principaux, dont les noms apparaissent en tête de chapitre. Les destins individuels sont ainsi morcelés, interrompus par les autres types de chapitres, dans un procédé proche du montage alterné cinématographique (l'histoire de certains personnages est parfois poursuivie dans un chapitre portant le nom d'un autre personnage). Cette structure revêt à la fois une fonction didactique (la chapitration crée un fil conducteur dans une narration discontinue) et une fonction esthétique (ce morcellement représente aussi la discontinuité et la complexité des trajectoires sociales des personnages).

6 Le deuxième type de chapitres est intitulé « Newsreel » ("Actualités »); les chapitres sont numérotés de I à LXVII, en continu du début de 42nd Parallel à la fin de The Big Money. Ils se composent de fragments issus de la presse contemporaine, de slogans, de 
passages de chansons populaires, etc. La variété des sources collées, accumulées tout au long de la trilogie, crée un magma unique de bribes de texte : la numérotation continue de ces chapitres et la présence récurrente de ce matériau textuel fragmenté contribuent à donner son unité à U.S.A. Le collage y est rendu visible par les différentes typographies, qui signalent la diversité des sources et évoquent visuellement le processus de prélèvement et de réagencement. Cette pluralité des typographies manifeste la co-présence des événements sans hiérarchie : un fait divers local sera mis sur le même plan qu'une chanson populaire et qu'un évènement politique majeur. Le collage permet ainsi de donner à voir, autant qu'à lire, la simultanéité des événements, voire leur contradiction, et donc de retranscrire la relativité du réel.

7 "The Camera Eye » est le troisième type de chapitres. Ils sont également numérotés en continu dans toute la trilogie, en chiffres arabes, de 1 à 51 . Il s'agit de récits en stream of consciousness : sans aucune ponctuation, ces textes se présentent comme le monologue intérieur d'une conscience saisissant le réel de manière à la fois pluriperceptive et fragmentée. Centrés sur les émotions et les sensations d'un sujet, les chapitres «Camera Eye » peuvent être considérés comme autobiographiques (Morel 1998 : 40).

Le dernier type de chapitres consiste en des biographies, c'est-à-dire des portraits de personnages historiques contemporains. Leurs titres font la plupart du temps référence à un trait particulier de la personnalité centrale, qui est elle-même en lien avec le parcours des personnages : par exemple Eugene Debs, un des fondateurs du mouvement ouvrier Industrial Workers of the World dont plusieurs personnages font partie, fait l'objet d'une biographie intitulée «Lover of Mankind» (Dos Passos [1938] 1996 : 30). De ces portraits, Dos Passos déclare à The Paris Review (Sanders 1969) :

C'est extrêmement difficile de dire comment j'en suis venu à ajouter ces portraits. J'essayais de saisir différentes facettes de mon sujet, d'obtenir quelque chose d'un peu plus précis que la fiction, et en même temps de travailler ces textes à l'intérieur de la représentation fictionnelle. Le but était toujours de produire de la fiction. C'est pourquoi j'étais totalement incapable de comprendre la dichotomie fiction/ non-fiction. J'étais comme sur la ligne qui les sépare, me déplaçant très rapidement d'un champ à l'autre ${ }^{1}$.

En effet, ce "dispositif narratif à quatre entrées » (Morel 1996 : 228), où des extraits de presse et des biographies de personnalités publiques sont articulés à la diégèse, offre une vue panoramique sur la réalité sociale, entre fiction et non-fiction. La continuité des chapitres supplante alors l'unité des trois romans, qui font en réalité office de supra-chapitration. De plus, les événements relatés dans la diégèse peuvent se trouver évoqués dans un collage de "Newsreel » ou dans une biographie : les quatre types de chapitres se révèlent donc poreux, connectés les uns aux autres, dans un processus d'échos qui dépasse la clôture canonique des chapitres et des romans.

\section{William S. Burroughs : le cut-up ou le roman pulvérisé}

9 La trilogie de William Burroughs procède du même effacement des limites du roman, suivant d'autres modalités. Composée de The Soft Machine (1961, dernière version publiée 1967), The Ticket That Exploded (1962, dernière version publiée 1968) et Nova Express (1964), elle repose sur le principe du cut-up, procédé inventé par Burroughs et le plasticien Brion Gysin : il consiste à découper de manière systématique deux textes au moins et à les ré-agencer suivant un ordre prédéterminé pour composer un nouveau texte. Le texte ainsi créé peut à son tour faire l'objet d'un nouveau découpage et d'une 
nouvelle combinaison à un autre texte: le cut-up repose ainsi sur un principe d' autogénération (Batt 1975 : 142).

La prose fragmentée de la trilogie relate une guerre interplanétaire entre le Complot Nova, organisation cherchant à asservir les individus par la diffusion de messages et d'images, et des groupes de résistants qui tentent de mettre fin à cette domination en coupant les lignes de diffusion de ces messages, c'est-à-dire en utilisant le cut-up. L'extrait suivant donne à lire un appel des résistants à s'insurger contre les Livres du Conseils, un des organes utilisés par le Complot Nova pour maintenir son emprise sur la population: "Towers, open fire - Explode word lines of the earth-Combat troops show board books and dictate out symbol language of virus enemy - Fight, controlled body prisoners - Cut all tape ${ }^{2} »$ (Burroughs [1962, 1967] 1994 : 104).

11 Le cut-up permet de réagencer un chapitre pour en créer un nouveau dans un autre roman: s'opère alors un transfert de fragments qui crée des connexions entre les différents chapitres mais aussi entre les romans. En connectant ainsi plusieurs espaces du texte, le cut-up contrecarre la linéarité constitutive de l'écrit. C'est par exemple le cas des chapitres «Gongs of Violence " dans The Soft Machine, "Combat troops in the area » dans The Ticket That Exploded, et du sous-chapitre "Towers open Fire», dans le chapitre "Chinese Laundry » de Nova Express, qui sont constitués du même matériau textuel. Ces mêmes fragments composent également la bande sonore du film Towers Open Fire, écrit par Burroughs et réalisé par Anthony Balch (1963), et apparaissent encore dans un enregistrement appelé "Uranian Willy ", qui figure sur le disque de cut-ups sonores, Call me Burroughs (1965). Le cut-up fait ainsi déborder le texte de toutes parts : il déborde d'abord la page, parce que celle-ci peut faire l'objet de nombreux découpages et recompositions; il déborde ensuite le chapitre en tant qu'unité narrative, puisque chaque chapitre peut être le réagencement des précédents; il déborde aussi le roman, dans la mesure où les cut-ups s'affranchissent des limites du livre, et il déborde même l'écrit : plusieurs groupes fragments ont en effet été utilisés dans des enregistrements sonores et des films.

Le cut-up apparaît alors comme une structure fractale: les fragments, chapitres, romans, voire l'ensemble de l'œuvre de Burroughs, sont régis par la même opération de morcellement-composition-redécoupage-recomposition - à tous les niveaux, de la plus petite phrase à l'ensemble du projet burroughsien. Ainsi, « la subdivision en chapitres n'a maintenant qu'une fonction résiduelle et minime - pour ainsi dire uniquement typographique - de scansion approximative de la matière informe et rutilante " (Amoruso 1975 : 139). Beaucoup plus qu'un collage, la trilogie Nova est un collage de collages, ou encore un montage kaléidoscopique: chaque fragment est l'entité nucléaire d'un système dont la fragmentation se répercute à tous les niveaux de l'œuvre, voire à l'extérieur de celle-ci.

\section{Ballard : le montage à l'ère de la simulation}

The Atrocity Exhibition de James Graham Ballard ne présente pas non plus de structure linéaire, ni de diégèse organisée : il s'agirait davantage d'une série de tableaux ou de scènes (au sens cinématographique), dont les liens sont parfois explicites, parfois beaucoup plus vagues. En effet, le texte est composé d'un montage de courts paragraphes, regroupés en quinze chapitres, chacun suivi d'une série de notes. Neuf de ces chapitres sont eux-mêmes subdivisés en paragraphes titrés (à la manière d'une 
sous-chapitration), et quatre sont construits en montage alterné : une longue phrase en italiques est interrompue à intervalles réguliers par des paragraphes composant euxmêmes un texte à part entière. À la fin de l'édition de 1990, quatre appendices ont été ajoutés : il s'agit de textes autonomes sans lien avec les chapitres précédents.

La trame romanesque principale se déroule dans un institut scientifique où sont menées des expériences de réalité virtuelle: un personnage central entreprend de reproduire virtuellement des événements médiatiques (l'assassinat de Kennedy, le suicide de Marilyn Monroe, le bombardement d'Hiroshima, la guerre du Vietnam). Il fabrique également des projections géantes de corps de stars et mène des expérimentations sur le lien entre les perversions sexuelles et les images. L'identité du protagoniste est floue: tantôt présenté comme patient de l'institut, tantôt comme chercheur, son nom change à chaque chapitre, dans un jeu de langage proche du métagramme. Appelé successivement Travis, Talbot, Traven, Tallis, Trabert, Talbert, Travers, il est parfois seulement désigné par le pronom «il ${ }^{3}$ ». L'instabilité des repères spatio-temporels et l'indifférenciation entre les événements vécus et les fantasmes du protagoniste plongent le lecteur dans un univers instable, une sorte de simulation où s'effacent les limites du réel et du virtuel.

Cette instabilité provient notamment du fait que les chapitres composant l'ouvrage ont d'abord été publiés séparément en revue, puis en un "roman » en 1972, lui-même réédité en 1990, augmenté de notes et d'une préface de William Burroughs. Il ne s'agit pas pour autant d'une suite de nouvelles puisqu'il existe bel et bien une continuité entre les textes-chapitres (notamment par la présence de personnages récurrents) et même une forme d'intrigue, dans la mesure où la lecture successive des chapitres permet l'élucidation progressive de certaines problématiques (on comprend notamment au fur et à mesure quel est le projet du personnage principal).

Les liens entre les paragraphes qui composent chaque chapitre peuvent être ténus (brusques changements de lieu, personnages désignés uniquement par des pronoms, écriture fragmentée), ou plus explicites. Les titres des paragraphes et des chapitres reprennent des expressions issues du corps du texte (parfois dans le même paragraphe, parfois dans un autre chapitre): comme dans les trilogies de Dos Passos et de Burroughs, le lecteur est conduit à établir des connexions entre des espaces distincts du roman et donc à tendre à une lecture non linéaire.

17 La présence de notes exacerbe cette rupture de la linéarité. Leur contenu est varié : si elles servent souvent à expliciter le texte principal en apportant des précisions sur la diégèse, elles relèvent également de l'anecdote, voire de l'autobiographie, sur un ton volontiers ironique. Avec ces notes, le texte fait retour sur lui-même à plusieurs titres : sur le plan sémantique, les notes expliquent le texte principal, enrichissent ou éclairent son sens; sur le plan temporel, texte et péritexte font cohabiter deux temporalités différentes, celle de la création et celle du commentaire; enfin, sur le plan du sens de lecture, le texte et la note se renvoient l'un à l'autre, amenant à une lecture fragmentée par les allers-retours entre les deux espaces textuels. Dans la préface du roman, Ballard invite explicitement le lecteur à cette lecture non linéaire $(2006: 6)$ :

Readers who find themselves daunted by the unfamiliar narrative structure of The Atrocity Exhibition - far simpler than it seems at first glance - might try a different approach. Rather than start at the beginning of each chapter, as in a conventional novel, simply turn the pages until a paragraph catches your eye. If the ideas or images seem interesting, scan nearby paragraphs for anything that resonates in an 
intriguing way. Fairly soon, I hope, the fog will clear, and the underlying narrative

will reveal itself. In effect, you will be reading the book in the way it was written ${ }^{4}$. disait qu'on pouvait lire ses livres «à partir de n'importe quel point d'interrogation " (Gysin 1975 : 188-189), le texte ballardien modifie le sens de la lecture. Dans les trois œuvres, la fonction structurante du chapitre, qui introduit habituellement des pauses et des ruptures dans la continuité romanesque, s'effrite au profit de connexions différentes, créées par le collage et le montage. En contrecarrant la successivité de l'écrit, les trois auteurs poursuivent également un objectif critique de déconstruction des discours tout faits, plus particulièrement ceux issus des médias de masse.

\section{La chapitration comme moyen d'interroger les médias}

\section{Dos Passos : mettre au jour la construction du discours médiatique}

Dans U.S.A., les journaux que lisent les personnages sont fréquemment évoqués et font partie intégrante de leur parcours : la presse y est souvent présentée comme capable de modeler leur opinion et d'influencer leurs décisions. Le pouvoir de la presse dans la diégèse se heurte aux fragments de journaux dans les "Newsreels »: l'emprise idéologique du discours médiatique y est comme passée au broyeur. En effet, le collage permet de déplier les procédés d'agencement et de mise en scène de l'information : en découpant et en ré-agençant les extraits, l'auteur met en lumière la stéréotypie des titres, les amalgames, l'accumulation d'informations ou leur redondance (Morel 1978 : 68). Dos Passos emploie le collage pour défaire ce que la presse a fait, pour le rendre lisible et, sans qu'un quelconque narrateur n'ait à intervenir, pour que le lecteur adopte une posture critique face à l'information.

Les «Newsreels » représentent également un passage hors de la fiction: en insérant dans le texte ces éléments extra-littéraires, Dos Passos signale constamment une extériorité qui renforce le pouvoir critique du collage. En faisant entrer la presse et le réel dans sa trilogie, l'auteur permet une double mise à distance critique des médias : d'une part, les «Newsreels » permettent d'interroger la trajectoire idéologique des personnages (leurs lectures engagées étant potentiellement broyées dans ces chapitres), et d'autre part, c'est aussi le rapport à la presse du lecteur lui-même qui est mis en jeu. Cette fonction quasi didactique du collage prend un tour beaucoup plus virulent chez William Burroughs, qui entendait faire du cut-up une méthode de guérilla langagière.

\section{Burroughs : détruire les lignes de communication du pouvoir}

21 La trilogie Nova est une dystopie dans laquelle l'univers est contrôlé par des médias malfaisants. Les images et le langage sont devenus des moyens d'asservissement des individus, qui sont tenus dans un état de dépendance et enfermés dans une réalité truquée : le Reality Studio produit en continu un reality film, substitut de réalité pour les intoxiqués. Le seul moyen pour lutter contre cette addiction est d'employer le cut-up pour couper les lignes de communication de ce film.

La technique du cut-up est en quelque sorte un pharmakon, à la fois poison et remède : il est tout autant une manifestation du chaos pour le lecteur qui plonge dans le tumulte 
des fragments, qu'un remède à l'intoxication pour les personnages enfermés dans le reality film. La trilogie est ainsi jalonnée d'appels à couper les lignes de mots et d'images à mesure que le lecteur lit le texte fragmenté par cette même technique. La duplicité du cut-up révèle ainsi son caractère performatif : le cut-up est en même temps un motif dans la fiction et le moyen de créer cette fiction. Le texte de la trilogie revêt ainsi un double statut (Batt 1975) : il est à la fois un texte-représentation (la diégèse) et un texte-action (où le cut-up se fait comme en temps réel). Or la seule solution pour vaincre la Conspiration Nova est de passer au niveau du texte-action. Comme l'explique Noëlle Batt (1975 : 63) :

ce conflit d'esprit révolutionnaire est résolument non dialectique tant qu'il s'exprime au niveau de la représentation. Condamné à l'improductivité totale, il ne peut que se reproduire à intervalles réguliers selon un schéma inchangé. Il faut pour voir apparaître un mouvement dialectique passer sur un autre plan, celui de l'écriture [...].

La déconstruction des chapitres, qui conduit à une lecture non linéaire, permet ce passage sur le plan du texte-action, c'est-à-dire de la matière-texte: le discours médiatique asservissant de la Conspiration Nova est alors détruit.

\section{Ballard : la critique de l'image-simulation}

Ballard, plus tard dans le siècle, entreprend aussi de déconstruire le pouvoir hypnotisant des médias. The Atrocity Exhibition représente « le modèle actuel de cette science-fiction qui n'en est plus une » (Baudrillard 1981 : 183), en dépeignant un monde envahi par les simulations, qui ne sont pas des fictions, mais des substituts de réalité. L'auteur explicite cette inquiétude face à l'omniprésence des images dans une note du chapitre 10, «Plan for the Assassination of Jacqueline Kennedy » ([1990] 2006 : 145):

The media landscape of the present day is a map in search of territory. A huge volume of sensational and often toxic imagery inundates our minds, much of fictional in content. How do we make sense of this ceaseless flow of advertising and publicity, news and entertainment, where presidential campaigns and moon voyages are presented in terms indistinguishable from the launch of a new candy bar or deodorant? ${ }^{5}$

Ballard déstructure et déhiérarchise le texte romanesque en une multitude de scènes indépendantes et interconnectées à la fois : en faisant de chaque texte une image, il montre que tout est image, de la même manière que ses personnages vivent dans un environnement dominé par les simulations. Comme chez Burroughs, la déconstruction du roman prend un tour performatif: le devenir-image des paragraphes se fait en même temps que ces images sont évoquées dans la diégèse. En effet, «T.» recrée des faits réels sous forme de simulations, à la manière d'un Reality Studio de Burroughs : son obsession pour les événements médiatiques est telle qu'elle semble contaminer la matière-texte elle-même.

La structure des chapitres, rendue poreuse et instable par l'intervention du collagemontage, permet donc aux trois auteurs de mettre le texte en action et de porter un discours critique sur les médias de leur temps. Dos Passos utilise un authentique matériau textuel issu de la presse pour interroger son pouvoir; Burroughs dénonce la fausseté même du langage articulé, son caractère aliénant, et invite à le broyer ; Ballard exhibe la fabrique des images médiatiques, faisant entrer le lecteur dans l'obsession du personnage principal. On voit ainsi se dessiner une dimension politique du chapitre : la 
linéarité de la lecture renvoie à un ordre social que les auteurs entendent questionner, voire renverser.

\section{De la dimension politique du chapitre}

Les thèmes politiques sont omniprésents dans les trois œuvres: Dos Passos évoque largement la lutte sociale et l'engagement syndical, Burroughs met en scène une insurrection interplanétaire tout au long de sa trilogie, et le traitement médiatique des événements politiques, comme la guerre du Vietnam, la conquête spatiale ou l'assassinat de JFK, occupe une large place dans le roman de Ballard.

Dès lors, et compte tenu de la critique des médias de masse que ces auteurs livrent par le truchement du découpage en chapitres, ne peut-on pas considérer qu'il existe, dans ces œuvres et peut-être de manière plus globale, une politique du chapitre ? Le courtcircuit constant qu'opèrent les procédés de collage-montage vis-à-vis de la chapitration aurait ainsi pour fonction de révéler la dimension idéologique de tout agencement du texte.

\section{Dos Passos : dresser un panorama social}

Nous l'avons vu, dans U.S.A., Dos Passos poursuit un objectif de définition panoramique de la société américaine de son temps. Ce projet est annoncé dans l'introduction aux trois romans de la trilogie, rédigée lors de la parution en un volume ([1938] $1996: 2-3)$ :

U.S.A. is the slice of a continent. U.S.A. is a group of holding companies, some aggregations of trade unions, a set of laws bound in calf, a radio network, a chain of moving picture theatres, a column of stock quotations rubbed out and written in by a Western Union boy on a blackboard, a public library full of old newspapers and dogeared history books with protests scrawled on the margins in pencil. U.S.A. is the world's greatest river valley fringed with mountains and hills, U.S.A. is a set of bigmouthed officials with too many bank accounts. U.S.A. is a lot of men buried in their uniforms in Arlington Cemetery. U.S.A. is the letters at the end of an address when you are away from home. But mostly U.S.A. is the speech of people . $^{6}$

D'emblée le projet romanesque de Dos Passos s'ancre dans la réalité polymorphe des États-Unis : l'accumulation de définitions combinée à l'anaphore «U.S.A. is " permet de transcrire l'hétérogénéité, voire les contradictions de l'espace social étasunien. Cette énumération en préambule de la trilogie signale la multilinéarité du texte et de la chapitration : pour rendre compte de la diversité - et des disparités - de la société américaine, une structure non linéaire s'impose.

En effet, le montage des différents types de chapitres permet l'articulation de formes hétérogènes; chacune de ces formes est centrée sur un type de relation de l'individu à la société : il s'agit d'une expérience du monde totalement intériorisée et intime dans "The Camera Eye»; les chapitres centrés sur les personnages mettent en avant les rapports de classe entre les individus; les biographies introduisent un rapport historique, voire mythologique, entre le lecteur et les personnalités publiques ; enfin, les « Newsreels » lui permettent d'adopter un rapport distancié au discours tenu par les médias sur les événements contemporains. Comme nous l'avons évoqué plus haut, il existe une porosité entre les différents types de chapitres : cette ouverture des quatre espaces narratifs les uns sur les autres a pour fonction de présenter une expérience collective ; en cela, la chapitration revêt une dimension politique. 
29 Mais plus profondément, l'emploi du montage chez Dos Passos «vise à délier [...] le rapport du genre romanesque à l'idéologie bourgeoise, tel qu'il s'est noué au XIX siècle» (Morel 1978: 67). L'ordre du discours, matérialisé par une chapitration linéaire, coïnciderait ainsi avec l'ordre social : la structure de U.S.A., en tissant entre les différents types de chapitres des liens obliques, aurait pour fonction de défaire le discours bourgeois, c'est-à-dire le discours dominant et univoque, pour permettre au "speech of people", aux paroles des gens, au foisonnement des voix du peuple, de faire leur entrée dans l'espace du roman.

\section{Burroughs : s'émanciper du sens de la lecture}

Pour Burroughs, la politique du chapitre s'inscrit dans une attaque en règle contre le Livre comme instrument de domination (livre religieux ou texte de lois) : en effet, dans sa trilogie, les «board books » (« Les Livres du Conseil » dans la traduction de C. Pélieu) contribuent, avec le Reality Studio, au maintien de l'intoxication des individus et «constituent l'organe officiel où se concrétisent les possibilités contrôlantes du Verbe » (Batt $1975: 40$ ). Mettre fin à la linéarité du livre et à l'unité du chapitre procède alors d'une déconstruction du langage et de sa métaphysique (Burroughs est assez proche en ce sens de la pensée de Derrida) : il s'agit bien d'abolir le Verbe premier qui fonde le Livre, l'ordre et la loi.

31 Tout au long de la trilogie, le lecteur est invité à utiliser le cut-up et à passer sur le plan du texte-action. En outre, Burroughs est aussi l'auteur de plusieurs manifestes (The Cutup Method of Brion Gysin, Invisible Generation et Electronic Revolution) dans lesquels cette injonction à couper les messages s'applique à la vie quotidienne. Il s'agit ici à la fois de libérer la lecture de sa linéarité et de libérer le lecteur de son conditionnement. En ce sens, l'emploi du collage par William Burroughs pose la question de la finalité de l'organisation du livre : elle serait selon lui vecteur d'un ordre idéologique.

Le cut-up et son entreprise de déconstruction du chapitre et du livre poussent le lecteur à interroger ses propres attentes quant à la lecture : attend-il du roman qu'il lui offre un ordre (dont le chapitre serait en quelque sorte la manifestation sensible) ou une émancipation de la pensée par le dépassement de la lecture linéaire? Les connexions multiples entre les différents chapitres et les différentes œuvres de Burroughs laissent en effet la possibilité de lire le texte dans différents ordres, et donc de se le réapproprier et de créer sa propre la lecture.

Dos Passos renonçait à la linéarité des chapitres pour faire émerger d'autres voix dans le texte: avec Burroughs, ces voix se brisent elles-mêmes en une multitude de fragments ne laissant au lecteur d'autre choix que de renoncer à la linéarité de la lecture, à l'ordre du récit comme vecteur d'ordre social. Quelques années plus tard, Ballard se fait l'écho d'une importante mutation épistémologique ; il n'est plus question d'ouvrir le texte à d'autres voix ni de déconstruire le langage : c'est la domination des images que le chapitre permet de mettre en lumière.

\section{Ballard : mettre au jour les mécanismes du spectacle}

"Ce que vous devez faire, c'est renoncer à lire plus d'un chapitre à la fois, et n'essayez pas de le lire comme une nouvelle ou un récit conventionnels ${ }^{7}$ ", déclare Ballard dans un entretien (Lewis 1991: 28). Ce conseil - ou cette injonction - témoigne d'une 
conception close du chapitre, pourtant paradoxalement ouvert à un péritexte sous la forme de notes. C'est que la démarche de Ballard s'inscrit dans la recherche d'une écriture par images, par plans, à la fois indépendants les uns des autres et connectés : la présence d'intertitres à chaque paragraphe fait de ceux-ci des tableaux mouvants, déstructurés, des scènes agencées par les fantasmes de «T. ». Burroughs, préfacier de l'édition augmentée, estime que l'écriture de Ballard est l'équivalent de ce que Robert Rauschenberg fait en art : "littéralement faire exploser l'image ${ }^{8}$ " (Ballard $2003: 12$ ). Dans certains paragraphes de The Atrocity Exhibition, la fragmentation du texte devient en effet un découpage d'images, dans ce que Ballard appelle une "écriture kinesthésique » (2006: 96-97) :

Elements of an Orgasm. (1) Her ungainly transit across the passenger seat through the nearside door; (2) the conjunction of aluminized gutter trim with the volume of her thighs; (3) the crushing of her left breast by the door pillar, its self-extension as she swung her legs on to the sandy floor; (4) the overlay of her knees and the metal door flank; (5) the ellipsoid erasure of dust as her hip brushed the nearside fender; (6) the hard transept of the door mechanism within the absolute erosion of the landscape; (7) her movements distorted in the projecting carapace of the radiator assembly; (8) the conjunction of her thighs with the arch of the motor bridge, the contrast of smooth epithelium and corrugated concrete; (9) her weak ankles in the soft ash; (10) the pressure of her right hand on the chromium trim of the inboard headlamp; (11) the forming a damp canopy in the cleavage of her blouse - the entire landscape expired within this irrigated trench; (12) the jut and rake of her pubis as she moved into the driving seat; (13) the junction of her thighs and the steering assembly; (14) the movements of her fingers across the chromium-tipped instrument heads'.

Le découpage de chaque chapitre en paragraphes titrés rend périlleuse l'identification des scènes: les actions des personnages ne se distinguent en rien de leurs fantasmes, pas plus que les événements des simulacres. C'est la réalité elle-même que Ballard interroge à travers la structure de son roman, comme il le déclare dans un entretien en 1977 (Barets et Frémion [1977] 2008 : 30) :

Le sens général de l'évolution qui conduit du roman classique à la $\mathrm{SF}$, c'est ce passage du réalisme à ce que j'appellerais du néo-réalisme. Ce n'est évidemment plus le réalisme au sens où aurait pu l'entendre Flaubert, et pourtant, pour moi, $L a$ Foire aux Atrocités est un livre profondément ancré dans cette nouvelle réalité. Je n'y parle que de ce qu'est notre vie. Que ce soit la télé, la pub, les communications, les vedettes, je les traite tous comme des éléments de notre réalité. Chacune de nos visions de la réalité se transforme à son tour en une part de la réalité sans qu'on ne puisse plus dérouler l'écheveau. [...] Car la plupart des éléments de notre vision de la réalité sont en fait fictifs, ce sont des éléments mythiques réifiés. Nous vivons dans un monde de simulacres.

Ainsi, chaque chapitre place le lecteur face à cette indétermination progressive du réel, car il ne peut connaître avec certitude le statut de ce qu'il lit, entre réalité et simulation. La chapitration de The Atrocity Exhibition, fondée sur la clôture des chapitres et des sous-chapitres transformés en images, dessine "cette nouvelle réalité »: un monde où simulation et réalité sont indifférenciables, c'est-à-dire un monde sans réalité. À la différence de Burroughs qui appelle à l'insurrection face à un ordre du langage aliénant, Ballard ne propose pas de solution mais dresse le constat implacable de la dissolution du réel dans un spectacle généralisé. 


\section{0-1990: une histoire culturelle de la déconstruction du chapitre}

De Dos Passos à Ballard se dessine une histoire du collage dans le domaine américain, qui semble inextricablement liée à la question toute politique de l'information et des médias. On peut noter en premier lieu la place de l'image cinématographique dans les trois œuvres, dont l'évocation apparaît de plus en plus inquiète : la culture populaire américaine, profondément innervée par les images d'Hollywood, de la publicité et de la télévision, se trouve disséquée par les opérations de collage et de montage des trois auteurs. U.S.A. est indéniablement influencé par le cinéma et la pratique du montage de Sergueï Eisenstein, que Dos Passos rencontre en 1928 lors d'un voyage en Union soviétique: l'auteur emploie le montage comme stratégie pour représenter le panorama social américain et voit dans le septième art une source d'innovation esthétique et sociale. Trois décennies plus tard, ce sont ces mêmes images que Burroughs entend combattre: le Reality Studio ne représente rien de moins que les images devenues aliénantes, substituts de réalité et nouvel opium du peuple. Ballard partage le constat burroughsien en faisant du lecteur un spectateur de ces imagessimulacres, et pointe avec ironie l'engouement pathologique du public pour le storytelling médiatique.

Les représentations frauduleuses (dans les médias, la presse, les images) prennent de l'ampleur tout au long du siècle, ce dont témoigne la généalogie Dos Passos-BurroughsBallard. Mais cette histoire dépasse le seul champ nord-américain : la confrontation entre la pratique du chapitre et l'usage du collage-montage renvoie plus globalement aux mutations de la culture occidentale, de la modernité à l'ère postmoderne. Lorsque Dos Passos déconstruit les discours de la presse pour mettre au jour ses artifices, lorsqu'il déstructure l'architecture romanesque, c'est dans la perspective toute moderniste d'une innovation esthétique aussi bien que d'un progrès social. Au tournant de la postmodernité, Burroughs l'amoderne renonce à la perspective d'une résolution des conflits de classe, sans abandonner le terrain politique (Murphy 1997): c'est pourquoi il place le conflit sur le plan du texte lui-même et pulvérise la chapitration pour en faire l'organe même d'un désordre libérateur. Ballard, quant à lui, illustre l'aplanissement des antagonismes propres à la postmodernité : à la fin des grands récits, le chapitre devient image et n'offre plus aucune perspective de résistance à la domination du spectacle généralisé. Ainsi, le montage et le collage révèlent la composante historico-culturelle du chapitre : en catalysant, dans la construction même de la narration, les changements de paradigmes épistémologiques qui s'opèrent tout au long du xxe siècle en Occident, Dos Passos, Burroughs et Ballard conduisent à penser le chapitre comme une émanation directe de son époque. 


\section{BIBLIOGRAPHIE}

Amoruso, Vito, 1975, « Le cut-up et la technique de la fugue ", dans Y. Le Pellec (dir.), Entretiens, Beat Generation, Rodez, Éditions Subervie, p. 133-141.

Aumont, Jacques, 1983, Esthétique du film, Paris, Nathan.

Ballard, James Graham, [1969-1990] 2003, La Foire aux atrocités, trad. François Rivière, Paris, Tristam.

Ballard, James Graham, [1969-1990] 2006, The Atrocity Exhibition, préface de William S. Burroughs, Londres, Harper Perennial.

Barets, Stan et Frémion, Yves, [1977] 2008, « Zones d'influence. Entretien avec J.G. Ballard », dans J. Schmidt et E. Notéris (dir.), J.G. Ballard, Hautes altitudes, Alfortville, Éditions è e, p. 27-37.

Batt, Noëlle, 1975, L'écriture de William Burroughs, thèse de troisième cycle d'Études nordaméricaines sous la direction de Pierre Dommergues, Saint-Denis, Université Paris 8.

Baudrillard, Jean, 1981, « Simulacres et science-fiction », dans Simulacres et simulation, Paris, Galilée.

Burroughs, William Seward, [1961, 1966] 1994, The Soft Machine, New York, Grove Press.

Burroughs, William Seward, [1962, 1967] 1994, The Ticket That Exploded, New York, Grove Press.

Burroughs, William Seward, [1964] 1994, Nova Express, New York, Grove Press.

Burroughs, William Seward, 1994, La Machine Molle, Le Ticket qui explosa, Nova Express, trad. Claude Pélieu et Mary Beach, Paris, Christian Bourgois.

Cran, Rona, 2014, Collage in Twentieth Century Art, Literature, and Culture, Farnham, Ashgate Publishing.

Dionne, Ugo, 2000, « Points de chute et points de fuite. Rupture chapitrale et ponctuation », dans J. Dürrenmatt (dir.), La Licorne, $\mathrm{n}^{\circ}$ 152, « La Ponctuation », p. 261-286.

Dos Passos, [1938] 2002, U.S.A : 42 ${ }^{e}$ Parallèle, trad. N. Guterman révisée par C. Jase ; L'An premier du siècle, trad. Y. Malartic révisée par C. Jase ; La Grosse galette, trad. de Charles de Richter révisée par Sabine Boulongne, préface Philippe Roger, Paris, Gallimard.

Dos Passos, John, [1938] 1996, U.S.A., New York, Library of America.

Dubois, Jacques, Dubois, Philippe, Édeline, François, Klinkenberg Jean-Marie et Minguet, Philippe, 1978, « Douze bribes pour décoller (en 40000 signes) », Revue d'esthétique - Collages, $\mathrm{n}^{\circ} 3 / 4$, p. 11-41.

Gysin, Brion, 1975, «Rub Out The Words », entretien avec Gérard-Georges Lemaire, dans Revue d'Esthétique - Il y a des poètes partout, $\mathrm{n}^{\circ}$ 3-4, p. 184-204.

Lewis, Jeremy, 1991, « An Interview with J. G. Ballard », Mississippi Review, vol. 20, n 1-2, p. 27-40. Morel, Jean-Pierre, 1978, « Montage, collage et discours romanesques dans les années vingt et trente ", dans D. Bablet (dir.), Collage et montage au théâtre et dans les autres arts, Lausanne, L’Âge d'homme, 1978, p. 38-73.

Morel, Jean-Pierre, 1996, « Jules Romains et Dos Passos », dans D. Viart (dir.), Jules Romains et les écritures de la simultanéité, Villeneuve d'Ascq, Presses Universitaires du Septentrion, p. 223-236. 
Morel, Jean-Pierre, 1998, Dos Passos. Multiplicité et solitude, Paris, Belin.

Murphy, Timothy S., 1997, Wising Up the Marks. The Amodern William S. Burroughs, Berkeley, University of California Press.

Sanders, David, 1969, « John Dos Passos, The Art of Fiction no. 44 », The Paris Review, $\mathrm{n}^{\circ}$ 46, [En ligne], http://www.theparisreview.org/interviews/4202/the-art-of-fiction-no-44-john-dospassos, consulté le 19 janvier 2019.

\section{NOTES}

1. «It's awfully hard to say how I came to add the portraits. I was trying to get different facets of my subject and trying to get something a little more accurate than fiction, at the same time to work these pieces into the fictional picture. The aim was always to produce fiction. That's why I was completely unable to understand the fictionnonfiction dichotomy. I was sort of on the edge between them, moving from one field to the other very rapidly $»$ (Nous traduisons).

2. «Tours, ouvrez le feu - Faites exploser les lignes-mots de la terre - Les troupes de combat montrent les Livres du Conseil et dictent le langage symbolique de l'ennemi virus - Combattez, vous les prisonniers contrôlés du corps - Coupez toutes les bandes »(Burroughs 1994 : 249).

3. Nous le désignerons par l'initiale « T. », dans la mesure où il s'agit bien d'un seul et même personnage.

4. «Au lieu de commencer chaque chapitre par son début, comme dans tout roman traditionnel, contentez-vous d'en tourner les pages jusqu'à ce qu'un paragraphe retienne votre attention. Si quelque idée ou quelque image vous y semble intéressante, balayez alors du regard les paragraphes voisins jusqu'à ce que vous y trouviez quelque chose qui résonne en vous de façon à piquer votre curiosité. Et bientôt, je l'espère, le rideau de brume se déchirera pour permettre au récit sous-jacent d'en émerger. À ce moment, vous lirez enfin ce livre exactement de la façon dont il a été écrit » (Ballard 2003 : 9-10).

5. «Le paysage médiatique d'aujourd'hui est une carte en quête de territoire. Un énorme volume d'imagerie sensationnelle et souvent toxique inonde nos esprits, et son contenu est pour la plus grande partie fictionnel. Comment dégageons-nous un quelconque sens de ce flux incessant de publicité et de réclame, de nouvelles et de divertissement, où les campagnes présidentielles et les voyages sur la lune sont présentés dans des termes qui ne les distinguent pas du lancement d'une nouvelle barre chocolatée ou d'un nouveau déodorant?» (Ballard 2003 : 165).

6. «Les USA, c'est une tranche de continent. Les USA, c'est un ensemble de sociétés de portefeuille, des regroupements de syndicats, une liasse de lois reliées en cuir, un réseau de radiodiffusion, une chaîne de salles de cinéma, une colonne de cotations boursières effacées et réécrites par un garçon de la Western Union sur un tableau noir, une bibliothèque publique pleine de vieux journaux et de manuels d'histoire éculés couverts de notes indignées griffonnées au crayon dans les marges. Les USA, c'est la plus grande vallée fluviale du monde, bordée de montagnes et de collines. Les USA, c'est une clique de fonctionnaires arrogants avec de trop nombreux comptes en banque. Les USA, ce sont beaucoup d'hommes enterrés en uniforme au cimetière d'Arlington. Les 
USA, ce sont les lettres à la fin d'une adresse lorsqu'on est loin de chez soi. Mais surtout, les USA, ce sont les paroles des gens » (Dos Passos [1938] 2002 : 35).

7. « What you have got to do is not read more than a chapter at a time, and don't try to read it as if you are trying to read a conventional short story, or a conventional narrative » (Nous traduisons).

8. « literally blowing up the image » (Ballard $2006: 8$ ).

9. «Préceptes orgasmatiques. (1) Le transit disgracieux de la passagère se rapprochant de la portière ; (2) la conjonction d'une tige d'aluminium et du volume de ses cuisses ; (3) l'écrasement de son sein gauche contre le montant de la portière, son rétablissement lorsqu'elle lança ses jambes vers le sol sablonneux ; (4) la superposition de ses genoux et de la face métallique de la portière; (5) l'effacement ellipsoïdal de la poussière lorsque sa hanche effleura l'aile avant ; (6) le transept précis découpé par le mécanisme de la porte dans le paysage entièrement érodé ; (7) ses mouvements déformés sur la surface réfléchissante de la calandre; (8) la conjonction de la surface médiane de ses cuisses et de l'arche du pont routier, le contraste entre son doux épithélium et le béton rugueux; (9) ses chevilles fragiles dans le sable mou; (10) la pression de sa main droite sur la bordure chromée du phare avant ; (11) la sueur recouvrant la peau d'une pellicule humide dans l'échancrure de son corsage ; (12) la saillie et l'inclinaison de son pubis quand il se glissa sur le siège conducteur ; (13) la rencontre de ses cuisses et du volant ; (14) le mouvement de ses doigts sur les boutons chromés » (Ballard 2003 : 114).

\section{RÉSUMÉS}

Cet article s'attache à examiner les effets du collage et du montage littéraire sur le chapitre, à travers trois œuvres du domaine anglo-saxon : la trilogie U.S.A. de John Dos Passos (achevée en 1936), la trilogie Nova de William Burroughs, composée de The Soft Machine (1961), The Ticket That Exploded (1962) et Nova Express (1964), et le roman The Atrocity Exhibition de James Graham Ballard (dans son édition augmentée de 1990).

Le collage et le montage, procédés de fragmentation du texte littéraire, introduisent une nouvelle mise en continuité des chapitres. Il apparaît que cette déconstruction de la linéarité romanesque est un moyen de mettre en question la construction du discours médiatique : la presse, la télévision, le cinéma, leurs artifices et leur pouvoir sont au cœur des trois œuvres de ce corpus. Plus globalement, le collage et le montage comme procédés d'écriture romanesque ont aussi pour fonction de porter un regard critique sur les médias de leur époque. Se dessine ainsi une dimension politique du chapitre, où l'agencement du texte devient en lui-même un outil critique.

This study focuses on the effects of literary collage and montage on chapter, through three Anglo-saxon literary works: John Dos Passos' U.S.A. (1938), William Burroughs' trilogy, composed of The Soft Machine (1961), The Ticket That Exploded (1962) and Nova express (1964), and James Graham Ballard's The Atrocity Exhibition (in his 1990's augmented edition).

Collage and montage, two methods of fragmentation of literary text, make it possible to introduce continuity between chapters. It seems that this deconstruction of novel linearity is a way to question structure of media discourse: press, television, movies, their artifices and their 
power are at the heart of the three works of this corpus. Overall, collage and montage as process of novel writing are used as taking a critical look at contemporary media. So, a political dimension of chapter is taking shape, in which the structure of the text becomes a critical tool itself.

INDEX

Keywords : collage, montage, chapter, Dos Passos (John), Ballard (James Graham), Burroughs (William)

Mots-clés : collage, montage, chapitre, Dos Passos (John), Ballard (James Graham), Burroughs (William)

\section{AUTEUR}

\section{CLÉMENTINE HOUGUE}

Le Mans Université, 3L.AM / Université Sorbonne Nouvelle, CERC 